\title{
Protocatecheuic Acid Underlies the Antioxidant Activity Exhibited by Illicium verum Fruit
}

\begin{abstract}
Illicium verum Hook. F, (Star anise) belongs to the family Illiciacea. In traditional medicine, it is used for variety of ailments. The current study was aimed at investigating the antioxidant potential of $I$. verum (IV) constituents. Various extract, fractions and pure compounds of I. verum were explored for their antioxidant activity using DPPH (1,1-diphenyl-2 picrylhydrazyl) and deoxyribose sugar assays. The structure of pure compound was elucidated through spectral studies (UV, IR, EIMS and ${ }^{1} \mathrm{H}-\mathrm{NMR}$ data). In DPPH assay, the methanolic extract of $I$. verum displayed antioxidant activity with an IC50 of $61 \mu \mathrm{g} / \mathrm{ml}$. Among its various fractions, the ethyl acetate (IV-EA) appeared to be potent antioxidant with IC50 value of $18 \mu \mathrm{g} / \mathrm{ml}$ and its sub-fractions, the ethyl acetate soluble subfraction of ethyl acetate fraction (IV-EA-EA-S), was most potent with IC50 of $42 \mu \mathrm{g} / \mathrm{ml}$. Further fractionation led to potent sub-fraction i.e. Petroleum etherethyl acetate insoluble sub fraction (IV-EA-PE:EA-I) with an IC50 of $12 \mu \mathrm{g} / \mathrm{ml}$. The purified fraction 13 subsequently appeared to be most potent $(7 \mu \mathrm{g} / \mathrm{ml})$ which led to the isolation of 3, 4-dihydroxy benzoic acid (protocatechuic acid, [1] as active principle. In deoxyribose degradation assay, the IV-E, IV-EA and protocatechuic acid also demonstrated antioxidant activity with IC50 of approximately 2000, 1400 and $600 \mu \mathrm{g} / \mathrm{ml}$ respectively. In conclusion, the protocatechuic acid [1] residing in $I$. verum most probably underlies its antioxidant action.
\end{abstract}

Keywords: Illicium verum; Protocatechuic acid; Antioxidant; DNA degradation inhibitor

Abbreviations: ROS: Oxygen Reactive Species, CRC: Concentration Response Curves

\section{Introduction}

Illicium verum Hook. F, belongs to the family Illiciacea and is commonly known as Badian. It is indigenous to Southern China and its dried fruit is consumed as spice in the food and drink industry as it provides flavor and enhances the taste of food. It is used as a condiment for flavoring curries, confectionery and for pickling [1]. In traditional medicine $I$.verum has been used as aromatic, anti-spasmodic, carminative, diuretic, expectorant, stomachic as well as stimulant. It is also useful in colic, constipation, dysentery, flatulence and insomnia [2,3]. Nadkarni (1976) Anti-HIV constituents are also reported from the roots of the plant [4].

Free radicals are produced when the body converts food to energy and also generated during several biochemical reactions. More recently, oxygen-reactive species (ROS), in particular free radicals such as hydroxyl have been recognized to be involved in several pathological conditions [5,6]. Antioxidants stabilize polyunsaturated fatty acid in food and are also known to reduce the risk of many diseases including cancer, cardiovascular, inflammatory and neurological disorders [7]. Therefore, the search and characterization of efficacious and cost effective antioxidants from natural products with fewer side effects

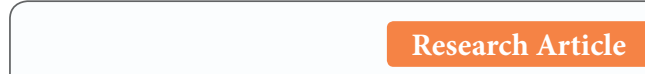

Volume 6 Issue 3 - 2017

Huma Aslam Bhatti ${ }^{2 *}$, Shagufta Khan ${ }^{1 *}$, Shaheen Faizi ${ }^{2}$, Ghulam Abbas ${ }^{3}$, Irfan $\mathrm{Ali}^{2}$, Saad Jawaid ${ }^{4}$, Anita Naushir Akbar Ali ${ }^{5}$, Rabia Jamy ${ }^{1}$, Fatima Shahid ${ }^{1}$, Muhammad Ali Versiani ${ }^{6}$ and Ahsana Dar ${ }^{2}$

${ }^{1}$ Department of Biological and Biomedical Sciences, Aga Khan University Hospital, Pakistan

${ }^{2} \mathrm{HEJ}$ Research Institute of Chemistry, University of Karachi, Pakistan

${ }^{3}$ Department of Pharmaceutical Sciences, COMSATS Institute of Information Technology, Pakistan

${ }^{4}$ Department of Emergency Medicine, King Fahad Medical City, Saudi Arabia

${ }^{5}$ Department of Anesthesiology, University of Arkansas for Medical Sciences, USA

${ }^{6}$ Department of Chemistry, Federal Urdu University of Arts, Pakistan

*Corresponding author: Huma Aslam Bhatti, HEJ Research Institute of Chemistry, International Center for Chemical and Biological Sciences, University of Karachi, Karachi-75270, Pakistan, Tel: +92-2199261779; Fax: +92-21-34819018, 99261713; Email: huma_alsam31@hotmail.com Shagufta Khan, Department of Biological and Biomedical Sciences, Aga Khan University Hospital, Medical College, Karachi-74800, Pakistan, Tel: (+92) 213-4864545; Fax: (+92) 213-493 4294, 493 2095; Email: shagufta.khan@aku.edu

Received: August 26, 2017 | Published: October 18, 2017

has been considerable interest in recent years. Previously, no investigation has been conducted regarding the antioxidant properties of any part of Illicium verum, including its fruit; however, there is a report in which antioxidant activities of herbs and species including $I$. verum from commercial sources were mentioned [8]. Therefore, the present study was conducted to evaluate the antioxidant activity of methanol extract of $I$. verum dried fruit via the bioassay guided fractionation using DPPH (1,1-diphenyl-2-picrylhydrazyl) and deoxyribose degradation assays. Rutin, ascorbic acid and trolox (vitamin E) were used as standard drugs in this study.

\section{Materials and Methods}

\section{Chemicals}

Rutin, vitamin C (L-ascorbic acid), trolox (analogue of vitamin E) and DPPH (1, 1-diphenyl-2 picrylhydrazyl) were purchased from Sigma (USA). Methanol was obtained from Merck, Germany. Silica gel $60 \mathrm{HF}_{254}$ was used for vacuum liquid chromatography. 


\section{Plant material}

Dried fruits of I. verum [5kg] were purchased from a local market and authenticated by Professor Dr. Anjum Perveen, Director Centre for Plant Conservation, Herbarium and Botanic Garden, University of Karachi. A voucher sample was submitted to Department of Biological and Biomedical Sciences Herbarium, Aga Khan University with reference No. IV-F-27-12-120.

\section{Bioassay guided extraction procedure}

Dried fruits of $I$. verum $(5 \mathrm{~kg})$ were extracted thrice with methanol at room temperature, and filtered after $48 \mathrm{hrs}$. The extracts were combined together and the solvent was removed under reduced pressure yielding a thick residue, $I$. verum extract (IV-E, $1287 \mathrm{~g})$. The I. verum extract $(788 \mathrm{~g})$ was partitioned between distilled water and petroleum ether to give petroleum ether and aqueous layers. The aqueous phase was extracted successively with ethyl acetate followed by butanol thrice and each layer was washed with water. The petroleum ether and butanol phases after evaporation of the solvent afforded petroleum ether (IV-PE, 65.88g) and butanol fractions (IV-But, $139.46 \mathrm{~g})$, respectively. The combined ethyl acetate phases were dried with anhydrous sodium sulphate and filtered, the filtrate on evaporation of solvent under reduced pressure furnished $32.57 \mathrm{~g}$ of ethyl acetate fraction (IV-EA) which was treated with petroleum ether to afford petroleum ether soluble (IV-EA-PE-S) and insoluble sub-fractions (IV-EA-PE-I), the latter was again divided into ethyl acetate soluble (IV-EA-EA-S) and insoluble subfractions (IV-EA-EA-I). The IV-EA-EA-S fraction (29.9 g), was taken in a small quantity of ethyl acetate and the resulting syrupy mass was poured into $700 \mathrm{ml}$ of petroleum ether with gentle shaking yielding petroleum ether-ethyl acetate soluble (IV-EA-PE:EA-S) and petroleum ether-ethyl acetate insoluble (IV-EA-PE:EA-I) sub-fractions. After freeze drying, the aqueous layer afforded aqueous fraction (IV-Aq, 220.6g), $50 \mathrm{~g}$ of which was treated with methanol affording methanol soluble (IV-Aq-M-S) and insoluble sub-fraction (IV-Aq-M-I) of IV-Aq. The IV-But was divided into ethyl acetate soluble (IV-But-EA-S) and insoluble (IV-But-EA-I) sub-fractions, respectively. The purified fraction, IV-EA-PE:EA-I $(17 \mathrm{~g})$ was subjected to vacuum liquid chromatography (VLC, silica gel, petroleum ether, ethyl acetate, methanol and water in order of increasing polarity) affording 38 fractions. Fraction 13G was divided into chloroform and chloroform: methanol (1:1) soluble fractions, 13G-C and 13G-CM respectively. 13G-C on further solvent separation gave chloroform soluble (13GC-C) and chloroform: methanol soluble fractions (13GC-M1 and 13GCM2). 13GC-M1 was subjected to PTLC which affording four bands of which band four was identified as 3,4-dihydroxybenzoic acid (protocatechuic acid), 1 (Figure 1) [9].

\section{Data analysis}

Results are expressed as the mean \pm SEM (n=number of observations). The $\mathrm{IC}_{50}$ values obtained graphically represented the concentration of sample required to scavenge $50 \%$ of free radicals or $50 \%$ inhibition of DNA damage by the hydroxyl radical. The median inhibitory concentration $\left(\mathrm{IC}_{50}\right.$ ) values with $95 \%$ confidence intervals. $p<0.05$ was considered significantly different using one-way ANOVA followed by Dunnett's test. The concentration-response curves (CRCs) were analyzed by non- linear regression using Graph PAD program (Graph PAD, San Diego, California, USA).

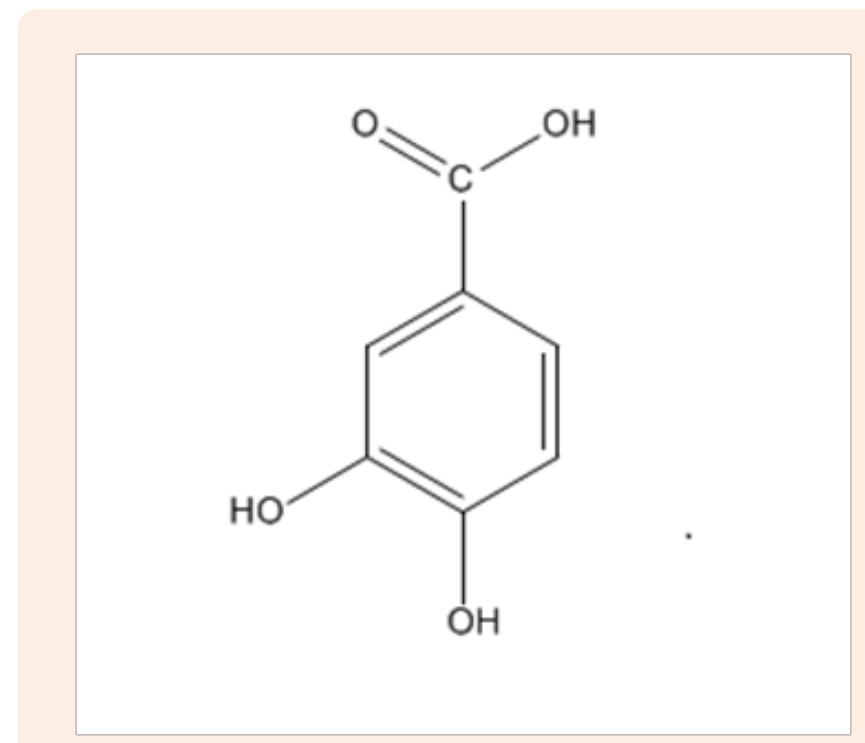

Figure 1: 3,4-dihydroxybenzoic acid.

\section{Results}

3,4-dihydroxybenzoic acid (protocatechuic acid, 1) was obtained as powder:

IR (KBr), $v \mathrm{~cm}^{-1} 3266,1674$ and 1296. UV (MeOH) $\lambda_{\max }(\mathrm{nm})$ 258. EIMS $(\mathrm{m} / \mathrm{z}) 154\left(\mathrm{M}^{+}\right)$.

${ }^{1} \mathrm{H}$ NMR (300MHz, $\left.\mathrm{C}_{3} \mathrm{D}_{3} \mathrm{O}\right): 7.52$ (d, $\left.J_{2,6}=2.0 \mathrm{~Hz}, \mathrm{H}-2\right), 7.46(\mathrm{dd}$, $\left.J_{5,6}=8.2 \mathrm{~Hz}, J_{2,6}=2.0 \mathrm{~Hz}, \mathrm{H} .6\right) 6.88\left(\mathrm{~d}, J_{5,6}=8.2 \mathrm{~Hz}, \mathrm{H}-5\right), 8.50$ (brs, aromatic $\mathrm{OH}$ ).

\section{DPPH assay}

Reaction mixture containing different concentration (0.5$200 \mu \mathrm{g} / \mathrm{ml}$ ) of the test sample (standard antioxidant, extract, fraction, sub-fractions, and pure compounds) in methanol and $300 \mu \mathrm{M}$ DPPH methanolic solutions were left at room temperature for a period of $30 \mathrm{~min}$. Absorbance was measured at $517 \mathrm{~nm}$ on a UV-VIS spectrophotometer and percent inhibition of DPPH free radicals after sample treatment was calculated [10].

\section{Deoxyribose degradation assay}

The hydroxyl radical induced damage to the deoxyribose (DNA) was determined in the absence of test compound (control) and presence of different concentrations of ascorbic acid $(100-2000 \mu \mathrm{g} /$ $\mathrm{ml})$, trolox $(100-500 \mu \mathrm{g} / \mathrm{ml})$, I. verum extract $(100-4000 \mu \mathrm{g} / \mathrm{ml})$, its ethyl acetate fraction $(100-4000 \mu \mathrm{g} / \mathrm{ml})$, compound $1(10$ $1000 \mu \mathrm{g} / \mathrm{ml}$ ), by the formation of thiobituturic active substances. Absorbance was measured spectrophotometrically at $532 \mathrm{~nm}$ and percent inhibition of DNA degradation after sample treatment was calculated as to compare control [11].

\section{Discussion}

The current study was aimed to investigate (bioassayguided fractionation) the antioxidant potential of I. verum using 
DPPH and deoxyribose degradation assay. Oxidative stress underlies the pathogenesis of several illnesses and antioxidants (natural and synthetic) helps in preventing it. However questions have been raised concerning the safety of some of the commercial antioxidants and linked with the mutagenesis and carcinogenesis [12]. Therefore, investigation and utilization of natural antioxidants as food product and medicine have been of considerable interest in recent years. It is well established that the DPPH assay provides an easy and rapid way to evaluate potential antioxidants [13]. Our data showed the methanolic extract of dried fruits of I. verum (IV-E) possess antioxidant activity $\left(\mathrm{IC}_{50} \sim 61 \mu \mathrm{g} /\right.$ $\mathrm{ml}$ ) exhibiting its possible role in prevention of oxidative stress (Table 1). In search of active principles, the IV-E was fractionated. Among all fractions, the ethyl acetate appeared to be most potent $\left(\mathrm{IC}_{50} \sim 18 \mu \mathrm{g} / \mathrm{ml}\right)$. The order of potency is trolox $>$ rutin $>$ ethyl acetate fraction $=$ ascorbic acid $>$ butanol faction $>$ extract thereby exhibiting that the antioxidant principles of the I. verum were most likely concentrated in the ethyl acetate phase (IV-EA).

Table 1: Effect of I. verum extract, its petroleum ether, butanol, ethyl acetate, aqueous fractions, ascorbic acid, rutin and trolox on percent antioxidant activity using DPPH assay.

\begin{tabular}{|c|c|c|c|c|c|c|c|}
\hline \multicolumn{8}{|c|}{ Dose $(\mu \mathrm{g} / \mathrm{ml})$} \\
\hline Sample & 3 & 6 & 12 & 25 & 50 & 75 & 100 \\
\hline IV-E & $03 \pm 1.9^{\text {n.s. }}$ & $07 \pm 1.2$ & $17 \pm 2.6$ & $24 \pm 4.3$ & $40 \pm 2.5$ & $58 \pm 6.2$ & $70 \pm 2.4$ \\
\hline IV-PE & $05 \pm 3.7^{\text {n.s. }}$ & $11 \pm 0.9$ & $12 \pm 1.4$ & $10 \pm 0.1$ & $13 \pm 1.3$ & $13 \pm 1.7$ & $14 \pm 2.5$ \\
\hline IV-But & $07 \pm 1.3$ & $16 \pm 3.1$ & $24 \pm 3.7$ & $35 \pm 6.0$ & $60 \pm 4.8$ & $82 \pm 4.8$ & $94 \pm 3.8$ \\
\hline IV-EA & $16 \pm 2.7$ & $30 \pm 3.6$ & $43 \pm 5.4$ & $63 \pm 5.8$ & $82 \pm 5.5$ & $91 \pm 1.3$ & $96 \pm 1.8$ \\
\hline IV-Aq & N.D. & N.D. & $07 \pm 1.1$ & $17 \pm 1.6$ & $24 \pm 1.1$ & $30 \pm 1.1$ & $29 \pm 1.3$ \\
\hline Ascorbic acid & $05 \pm 0.5$ & $11 \pm 1.7$ & $29 \pm 3.1$ & $63 \pm 2.7$ & $94 \pm 2.9$ & $96 \pm 1.4$ & N.D. \\
\hline Rutin & $24 \pm 2.3$ & $65 \pm 4.4$ & $88 \pm 4.6$ & $93 \pm 1.5$ & $96 \pm 1.7$ & N.D. & N.D. \\
\hline Trolox & $57 \pm 2.4$ & $93 \pm 2.1$ & $97 \pm 1.8$ & N.D. & N.D. & N.D. & N.D. \\
\hline
\end{tabular}

Each value represents mean $\pm \mathrm{SE}$ of five determinants, each in duplicate. Control absorbance $=0.8548 \mathrm{~nm} \pm 0.02(\mathrm{n}=35)$. N.D. $=$ Not done. All the values showed significant percent antioxidant activity $(\mathrm{p}<0.05)$ as compared to the control and n.s. represents non-significant difference.

IV-E, IV-E-PE, IV-But, IV-EA and IV-Aq represent I. verum extract, petroleum ether fraction, butanol fraction, ethyl acetate fraction and aqueous fraction, respectively.

The IV-EA was further treated with different solvents to obtain its various sub-fractions. Among various sub-fractions, the ethyl acetate soluble sub-fraction (IV-EA-EA-S) was most potent with $\mathrm{IC}_{50}$ of $42 \mu \mathrm{g} / \mathrm{ml}$ (Table 2). Further fractionation led to potent subfraction i.e. petroleum ether-ethyl acetate insoluble sub fraction (IV-EA-PE:EA-I) with an $\mathrm{IC}_{50}$ of $12 \mu \mathrm{g} / \mathrm{ml}$. The purified fraction 13 subsequently appeared to be most potent $(7 \mu \mathrm{g} / \mathrm{ml}$, (Table 3 ). Based on the aforementioned findings, purified fraction-13 (PF13) was further treated with different solvent system to obtain purified sub-fractions including PFS-13A, PFS-13B, PFS-13C, PFS-13D, PFS-13E, PFS-13F and PFS-13G. The fraction PFS-13A, which showed a predominant UV active spot on TLC (thin layer chromatography) was subjected to HPTLC (high performance TLC), furnishing four bands of which band 4P was identified as 3 , 4-dihydroxy benzoic (protocatechuic acid, 1, (Figure 1) through spectral studies. Regarding the profile of aromatic acids from $I$. verum as chemical constituents, identification of only $p$-hydroxy benzoic acid has been reported from this plant. It is important to note that 3,4-dihydroxy benzoic acid has been isolated from many plants e.g. Abies specie [14], Cinnamomum zeylanicum [15],
Delonix negia [16], Hibiscus sabdariffa [17], Musanga cecropioides [9], and Salvia miltiorrhiza [18], and its biological properties including antioxidant and antitumor activities have also been reported [19]. It is well established fact that superoxide radical and hydrogen peroxide are produced during aerobic metabolism and play vital role in physiological processes. The over production of hydroxyl radical may lead to tissue damage and degradation of important bio-molecules such as DNA by various mechanisms e.g. base pair mutations, deletions and insertions [20]. The damage to mitochondrial DNA could play a crucial role in pathogenesis of various disorders such as neurodegenerative diseases. Thus the scavenging and/or chelation of $\mathrm{Fe}^{+3}$ ions may provide important therapeutic significance against oxidative stress induced diseases [21]. Our data showed that in deoxyribose degradation assay, the IV-E, IV-EA and protocatechuic acid (1) also demonstrated antioxidant activity with $\mathrm{IC}_{50}$ of approximately 2000, 1400 and $600 \mu \mathrm{g} / \mathrm{ml}$ respectively (Table 4 ). The structures of protocatechuic acid were determined through UV, IR, Mass and ${ }^{1} \mathrm{H}$ NMR spectral studies (Figure 1), and comparison of the data with those reported in literature $[2,9,19,22,23]$. 
Table 2: Effect of sub-fractions (obtained from butanol, ethyl acetate, and aqueous fractions) on percent antioxidant activity using DPPH assay.

\begin{tabular}{|c|c|c|c|c|c|c|c|}
\hline \multicolumn{8}{|c|}{ Dose $(\mu \mathrm{g} / \mathrm{ml})$} \\
\hline Sample & 3 & 6 & 12 & 25 & 50 & 75 & 100 \\
\hline IV-But-EA-S & $4 \pm 0.8^{\mathrm{ns}}$ & $11 \pm 1.4$ & $18 \pm 2.1$ & $27 \pm 3.1$ & $52 \pm 2.9$ & $78 \pm 2.6$ & $79 \pm 5.2$ \\
\hline IV-But-EA-I & $7 \pm 1.3$ & $15 \pm 2.5$ & $26 \pm 1.9$ & $37 \pm 3.8$ & $70 \pm 4.1$ & $81 \pm 3.1$ & $80 \pm 2.7$ \\
\hline IV-EA-PE-S & $10 \pm 1.5$ & $09 \pm 1.1$ & $08 \pm 1.1$ & $10 \pm 2.4$ & $09 \pm 1.1$ & $11 \pm 1.5$ & $12 \pm 2.2$ \\
\hline IV-EA-PE-I & $9 \pm 1.2$ & $24 \pm 1.7$ & $38 \pm 4.1$ & $72 \pm 7.1$ & $93 \pm 9.2$ & $94 \pm 3.1$ & N.D. \\
\hline IV-EA-EA-S & $13 \pm 0.3$ & $27 \pm 2.6$ & $36 \pm 4.4$ & $88 \pm 0.8$ & $92 \pm 0.6$ & $94 \pm 0.3$ & N.D. \\
\hline IV-EA-EA-I & $16 \pm 1.3$ & $31 \pm 3.4$ & $54 \pm 6.4$ & $72 \pm 4.8$ & $88 \pm 3.3$ & $90 \pm 3.6$ & $92 \pm 3.2$ \\
\hline IV-EA-PE:EA-S & N.D. & $9 \pm 3.1$ & $16 \pm 1.2$ & $22 \pm 1.8$ & $32 \pm 0.7$ & $43 \pm 0.5$ & $56 \pm 1.6$ \\
\hline IV-EA-PE:EA-I & $14 \pm 1.1$ & $35 \pm 2.1$ & $56 \pm 2.5$ & $74 \pm 1.2$ & $75 \pm 1.1$ & $67 \pm 1.4$ & N.D. \\
\hline IV-Aq-M-S & N.D. & N.D. & $14 \pm 3.1$ & $13 \pm 3.7$ & $17 \pm 1.8$ & $19 \pm 3.2$ & $20 \pm 2.8$ \\
\hline IV-Aq-M-I & N.D. & N.D. & $14 \pm 4.1$ & $17 \pm 4.2$ & $13 \pm 3.4$ & $18 \pm 4.2$ & $17 \pm 2.9$ \\
\hline
\end{tabular}

Each value represents mean \pm SE of four determinants, each in duplicate. Control absorbance $=0.8392 \mathrm{~nm} \pm 0.02(\mathrm{n}=40)$. N.D. $=$ Not done. All the values showed significant percent antioxidant activity $(\mathrm{p}<0.05)$ as compared to the control and n.s. represents non-significant difference. IV-But-EA-S: Ethyl acetate soluble sub fraction of butanol fraction, IV-But-EA-I: Ethyl acetate insoluble sub fraction of the butanol fraction IV-EA-PE-S: Petroleum ether soluble sub-fraction of ethyl actate fraction, IV-EA-PE-I: Petroleum ether insoluble sub-fraction of ethyl actate fraction, IV-EA-EA-S: Ethyl acetate soluble sub-fraction of ethyl acetate fraction, IV-EA-EA-I: Ethyl acetate insoluble sub-fraction of ethyl acetate fraction, IV-EA-PE:EA-S: Petroleum ether, ethyl acetate soluble sub-fraction of ethyle acetate fraction, IV-EA-PE:EA-I: Petroleum ether, ethyl acetate insoluble sub-fraction of ethyle acetate fraction IVAq-M-S: Methanol soluble sub-fraction of aqueous fraction, IV-Aq-M-I: Methanol insoluble sub-fraction of aqueous fraction.

Table 3: The $\mathrm{IC}_{50}$ values of petroleum ether, ethyl acetate insolublesubfraction and its purified fractions on percent antioxidant activity using DPPH assay.

\begin{tabular}{|c|c|}
\hline Sample & IC50 \\
\hline IV-EA-PE:EA-I & $12 \pm 1.1$ \\
\hline Purified fraction 13 & $07 \pm 1.2$ \\
\hline Purified fraction group 1 & $19 \pm 0.4$ \\
\hline Purified fraction group 2 & $23 \pm 0.9$ \\
\hline Purified fraction group 3 & $29 \pm 0.6$ \\
\hline Purified fraction 23 and 25 & $38 \pm 0.5$ \\
\hline Purified fraction 26 and 24 & $45 \pm 0.3$ \\
\hline Purified fraction 37 & $54 \pm 2.1$ \\
\hline
\end{tabular}

Each value represents mean \pm SE of four determinants, each in duplicate. Control absorbance $=0.8756 \mathrm{~nm} \pm 0.03(\mathrm{n}=108)$. All the above mentioned $\mathrm{IC}_{50}$ values were found significantly different $(\mathrm{p}<0.05)$ from each other.

IV-EA-PE:EA-I: Petroleum ether, ethyl acetate insoluble sub-fraction of ethyle acetate fraction.

Purified fraction (PF) group-1 include PF-21, PF-22, PF-27, PF-33, PF-35, PF-36 and PF-38

Purified fraction group 2 include PF-10, PF-28, PF-29, PF-30 and PF-34.

Purified fraction group 3 include PF-11, PF-12PF-14, PF-15, PF-17, PF-18, PF-19, PF-20 and PF-32.
Table 4: Effect of $I$. velum extract, its ethyl acetate fractions, compound 1, ascorbic acid and trolox on percent inhibition of DNA degradation.

\begin{tabular}{|c|c|c|c|c|c|}
\hline & & \multicolumn{2}{|c|}{ Dose $(\boldsymbol{\mu g} / \mathbf{m l})$} & & \\
\hline Sample & $\mathbf{1 0 0}$ & $\mathbf{5 0 0}$ & $\mathbf{1 0 0 0}$ & $\mathbf{2 0 0 0}$ & $\mathbf{4 0 0 0}$ \\
\hline IV-E & $5 \pm 1.9$ n.s. & $08 \pm 2.1$ & $27 \pm 2.6$ & $54 \pm 5.1$ & $87 \pm 4.6$ \\
\hline IV-EA & $5 \pm 3.1$ n.s. & $19 \pm 4.4$ & $43 \pm 5.5$ & $77 \pm 4.9$ & $93 \pm 4.3$ \\
\hline Compound 1 & $16 \pm 2.7$ & $42 \pm 3.3$ & $91 \pm 2.6$ & N.D. & N.D. \\
\hline Ascorbic acid & $26 \pm 3.6$ & $63 \pm 3.2$ & $96 \pm 2.9$ & $95 \pm 4.1$ & N.D. \\
\hline Trolox & $41 \pm 4.2$ & $97 \pm 2.8$ & N.D. & N.D. & N.D. \\
\hline
\end{tabular}

Each value represents mean \pm SE of four determinants, each in duplicate. Control absorbance $=0.6979 n m \pm 0.02(n=24)$. N.D. $=$ Not done. All the values showed significant percent antioxidant activity $(\mathrm{p}<0.05)$ as compared to the control and n.s. represents non-significant difference.

\section{Conclusion}

The protocatechuic acid (1) residing in I. verum most probably underlies its antioxidant action. The present study provides the pharmacological basis for the use of Illicium verum fruits in preventing oxidative stress.

\section{Acknowledgement}

None. 


\section{Conflict of Interest}

None.

\section{References}

1. Yan Jh, Xiao XX, Huang KI (2002) Component analysis of volatile oil from Illicium verum hook. $f$. Journal of Central South University of Technology 9(3): 173-176.

2. Miyazawa M (1993) An Insecticidal Compound from Illicium verum ChemInform 24(44).

3. Chopra RN, Chopra IC, Varma B (1956) Glossary of Indian Medicinal Plants: Suppl. Council of Scientific \& Industrial Research.

4. Song WY, Ma YB, Bai X, Zhang XM, Gu Q, et al. (2007) Two new compounds and anti-HIV active constituents from Illicium verum. Planta Med 73(4): 372-375.

5. Bray TM (1999) Antioxidants and oxidative stress in health and disease: introduction. Proceedings of the Society for Experimental Biology and Medicine 222(3): 195-195.

6. Maxwell SR (1995) Prospects for the use of antioxidant therapies. Drugs 49(3): 345-361.

7. Dar A, Faizi S, Naqvi S, Roome T, Zikr-ur-Rehman S, et al. (2005) Analgesic and antioxidant activity of mangiferin and its derivatives: the structure activity relationship. Biol Pharm Bull 28(4): 596-600.

8. Liu X (2002) Antioxidant activities of herbs and spices from commercial sources. Shipin Kexue 23(1): 143.

9. Ayinde BA, Onwukaeme DN, Omogbai E (2007) Isolation and characterization of two phenolic compounds from the stem bark of Musanga cecropioides R. Brown (Moraceae). Acta Pol Pharm 64(2): 183-185.

10. Mensor LL, Menezes FS, Leitão GG, Reis AS, dos Santos TC, et al. (2001) Screening of Brazilian plant extracts for antioxidant activity by the use of DPPH free radical method. Phytother Res 15(2): 127130.

11. Wang L, Nishida H, Ogawa Y, Konishi T (2003) Prevention of oxidative injury in PC12 cells by a traditional Chinese medicine, Shengmai San, as a model of an antioxidant-based composite formula. Biol Pharm Bull 26(7): 1000-1004.
12. Subhasree B, Baskar R, Laxmi Keerthana R, Lijina Susan R, Rajasekaran P (2009) Evaluation of antioxidant potential in selected green leafy vegetables. Food chemistry 115(4): 1213-1220.

13. Duan XJ, Wei-Wei Zhang, Xiao-MingLi, Bin-Gui Wang (2006) Evaluation of antioxidant property of extract and fractions obtained from a red alga, Polysiphonia urceolata. Food chemistry 95(1): 3743.

14. Yang XW, Li SM, Shen YH, Zhang WD (2008) Phytochemical and biological studies of Abies species. Chem Biodivers 5(1): 56-81.

15. Jayaprakasha G, Ohnishi-Kameyama M, Ono H, Yoshida M, Jaganmohan Rao L, et al. (2006) Phenolic constituents in the fruits of Cinnamomum zeylanicum and their antioxidant activity. J Agric Food Chem 54(5): 1672-1679.

16. Chou CH, Leu LL (1992) Allelopathic substances and interactions of Delonix regia (Boj) Raf. J Chem Ecol 18(12): 2285-2303.

17. Lin WL, Hsieh YJ, Chou FP, Wang CJ, Cheng MT, et al. (2003) Hibiscus protocatechuic acid inhibits lipopolysaccharide-induced rat hepatic damage. Arch Toxicol 77(1): 42-47.

18. Zhu Y, Huang SH, Tan BK, Sun J, Whiteman M, etal. (2004) Antioxidants in Chinese herbal medicines: a biochemical perspective. Nat Prod Rep 21(4): 478-489.

19. Inayama S, Kenzo Harimaya, Hitoshi Hori, Tamiko Ohkura, Takeshi Kawamata, etal. (1984) Studies on non-sesquiterpenoid constituents of Gaillardia pulchella. II. Less lipophilic substances, methyl caffeate as an antitumor catecholic. Chemical and pharmaceutical bulletin 32(3): 1135-1141.

20. Wiseman H, Halliwell B (1996) Damage to DNA by reactive oxygen and nitrogen species: role in inflammatory disease and progression to cancer. Biochem J 313(Pt 1): 17-29.

21. Galey J (2001) Recent advances in the design of iron chelators against oxidative damage. Mini Rev Med Chem 1(3): 233-242.

22. Bianco A, Bonadies F, Melchioni C (2000) Synthesis of Regioselectively Protected Protocatechuic Acid Derivatives by Biomimetic Transformation of Quinic Acid. Molecules 5(10): 1094-1100.

23. Schuster B, Winter M, Herrmann K (1986) 4-O- $\beta$-D-Glucosides of Hydroxybenzoic and Hydroxycinnamic Acids-Their Synthesis and Determination in Berry Fruit and Vegetable. Zeitschrift für Naturforschung C 41(5-6): 511-520. 